\title{
Utilising the flexible generation potential of tidal range power plants to optimise economic value
}

\author{
Freddie Harcourt ${ }^{\mathrm{a}}$, Athanasios Angeloudis ${ }^{\mathrm{b}, \mathrm{c}, *}$, Matthew D. Piggott ${ }^{\mathrm{b}}$ \\ ${ }^{a}$ Energy Futures Lab, Imperial College London, UK \\ ${ }^{b}$ Department of Earth Science 85 Engineering, Imperial College London, UK \\ ${ }^{c}$ School of Engineering, Institute for Infrastructure $\&$ the Environment, The University of \\ Edinburgh, UK
}

\begin{abstract}
Tidal range renewable power plants have the capacity to deliver predictable energy to the electricity grid, subject to the known variability of the tides. Tidal power plants inherently feature advantages that characterise hydro-power more generally, including a lifetime exceeding alternative renewable energy technologies and relatively low Operation \& Maintenance costs. Nevertheless, the technology is typically inhibited by the significant upfront investment associated with capital costs. A key aspect that makes the technology stand out relative to other renewable options is the partial flexibility it possesses over the timing of power generation. In this study we provide details on a design methodology targeted at the optimisation of the temporal operation of a tidal range energy structure, specifically the Swansea Bay tidal lagoon that has been proposed within the Bristol Channel, UK. Apart from concentrating on the classical incentive of maximising energy, we formulate an objective functional in a manner that promotes the maximisation of income for the scheme from the Day-Ahead energy market. Simulation results demonstrate that there are opportunities to exploit the predictability of the tides and flexibility over the precise timing of power generation to incur a noticeable reduction in the subsidy costs that are often negotiated with regulators and governments. Additionally, we suggest that this approach should enable tidal range energy to play a more active role in ensuring security of supply in the UK. This is accentuated by the income-based optimisation controls that deliver on average more power over periods when demand is higher. For the Swansea Bay tidal lagoon case study a $23 \%$ increase is observed in the income obtained following the optimisation of its operation compared to a non-adaptive operation. Similarly, a $10 \%$ increase relative to an energy-maximisation approach over a year's operation suggests that simply maximising energy generation in a setting where power prices vary may not be an optimal strategy.
\end{abstract}

\footnotetext{
${ }^{*}$ Corresponding author

Email address: a.angeloudis@ed.ac.uk (Athanasios Angeloudis )
} 
Keywords: Tidal range energy, marine energy, resource assessment, income optimisation, flexible generation

\section{Introduction}

The differences between high and low tidal levels (i.e. the tidal range) in coastal locations represent a renewable energy source that has the advantage of being low-carbon and predictable. In particular, the theoretically available potential of tidal range energy in the UK has been estimated to be beyond the annual electricity demand of the entire country $(\approx 306 T W h)[1$. Considering a variety of technical and spatial constraints, a fraction of this resource can be practically captured by the construction of tidal power plants in areas where it becomes economically and environmentally viable [2]. Given this opportunity, it is important to understand the potential role of tidal range energy generation within the UK electricity system.

In areas with a high tidal range, impoundments can be built as barrages spanning entire estuarine basins, or as lagoons which can be positioned at the coastal boundaries of estuaries as well as fully offshore [3]. By separating the impounded area from the open sea, electricity may be generated from a head difference $(H)$, driven by tidal variations in the external water level and extracted by low-head hydro turbines [4. Tidal range generation is fundamentally different from other marine and offshore renewables as the duration of generation can be flexibly controlled and optimised alongside the energy conversion technology. Previous work [5] demonstrated the opportunity to extract more of the available resource through an adaptive operation where the hydraulic structure control in each tidal cycle was individually optimised.

Recent UK Government decisions [6] highlight the need for tidal range projects to demonstrate value for money in order to justify governmental support. We approach this challenge in two ways. Firstly, the concept of adaptive operation has been refined to further increase the predicted energy output, and thus decrease the cost per unit power generated. We achieve this by reformulating the objective function to take into account the effects of one tidal cycle's operation on a subsequent one, with a view to improve upon the relatively shortsighted approach that considered each tidal cycle individually [5. Secondly, the optimisation approach employed is generalised and applied to maximise the income a tidal range project receives from selling its power. This is achieved by utilising generation flexibility to help match the daily, weekly and seasonal trends in the value of electricity set by consumer demand and the merit order stack [7.

It has been noted previously that tidal range projects can potentially generate at times of high demand, or price, but outside the optimum window for energy output [8. Shaw and Watson 9] reported that initiating generation an hour earlier or later than the energetically optimum could still yield over $90 \%$ of the maximum energy output. This flexibility can be a useful tool in ensuring 
that energy demand is better matched, but there is little quantification in the literature of its value. As such, operational objectives can be altered so that plant income is maximized rather than the energy output. The latter has been the focus of several studies. For example, Aggidis and Benzon [10] examined energy output optimisation for an ebb-only generation barrage in the Mersey Estuary, UK. Yates et al. [11] conducted a series of operational modelling tests to examine potential increases in energy output through the use of pumping in tidal barrages. More recently, Angeloudis et al. [5] presented an energy optimisation framework for tidal range structures that couples gradient-based optimisation with operational models. In contrast, an income-based operation optimisation approach has not been previously documented with the exception of Merlin et al. 12] who proposed to address this challenge for the La Rance barrage in France through dynamic programming. As such, there is a lack of concrete evidence demonstrating the potential value in applying optimisation and modelling methods to exploit plant generation flexibility. This should be done both in the context of helping to make tidal power plant proposals (a) more financially competitive and (b) more valuable as constituent energy infrastructure assets.

The time-varying nature of the tides, coastal bathymetry and turbine characteristics necessitate numerical simulations to accurately estimate the electricity output from a given project. We apply the concept of "two-cycle" and income-based optimisation to operational models which are then used to inform hydrodynamics simulations capable of accounting for the impact of changing the operational objectives on both energy output and income [5. In turn, we discuss the implication of an optimised operation in the context of reducing the potential subsidy required in the early stages of the technology's deployment.

In contrast to earlier studies, the transferable methodology described here can be used to encourage power generation during time periods of greater energy demand. The methodology is demonstrated through an application to a practical case study of a tidal lagoon proposal in the UK. The optimised operation performance illustrates the complementary role tidal range energy can perform with intermittent energy technologies currently deployed at a large scale (e.g. offshore and onshore wind).

\section{Methodology}

\subsection{Tidal power plant operation}

The theoretical maximum energy that can be generated from a head difference $H$ was set out by Prandle [13] as:

$$
E_{\max }=\frac{1}{2} \rho g A H^{2},
$$

where $A$ is the plan surface area of the impounded water body, assumed above to be constant with depth, and with two physical parameters: the fluid density $\rho\left(\mathrm{kg} / \mathrm{m}^{3}\right)$ and the gravitational acceleration $g\left(\right.$ in $\left.\mathrm{m} / \mathrm{s}^{2}\right)$. In practice, the 
extractable energy from a tidal power plant in each tidal cycle is additionally related to (a) the installed turbine technology capabilities, (b) the spring-neap (and longer period) tidal and plan surface area variations at the site and (c) the design of the structure and its interaction with local and far-field hydrodynamics.

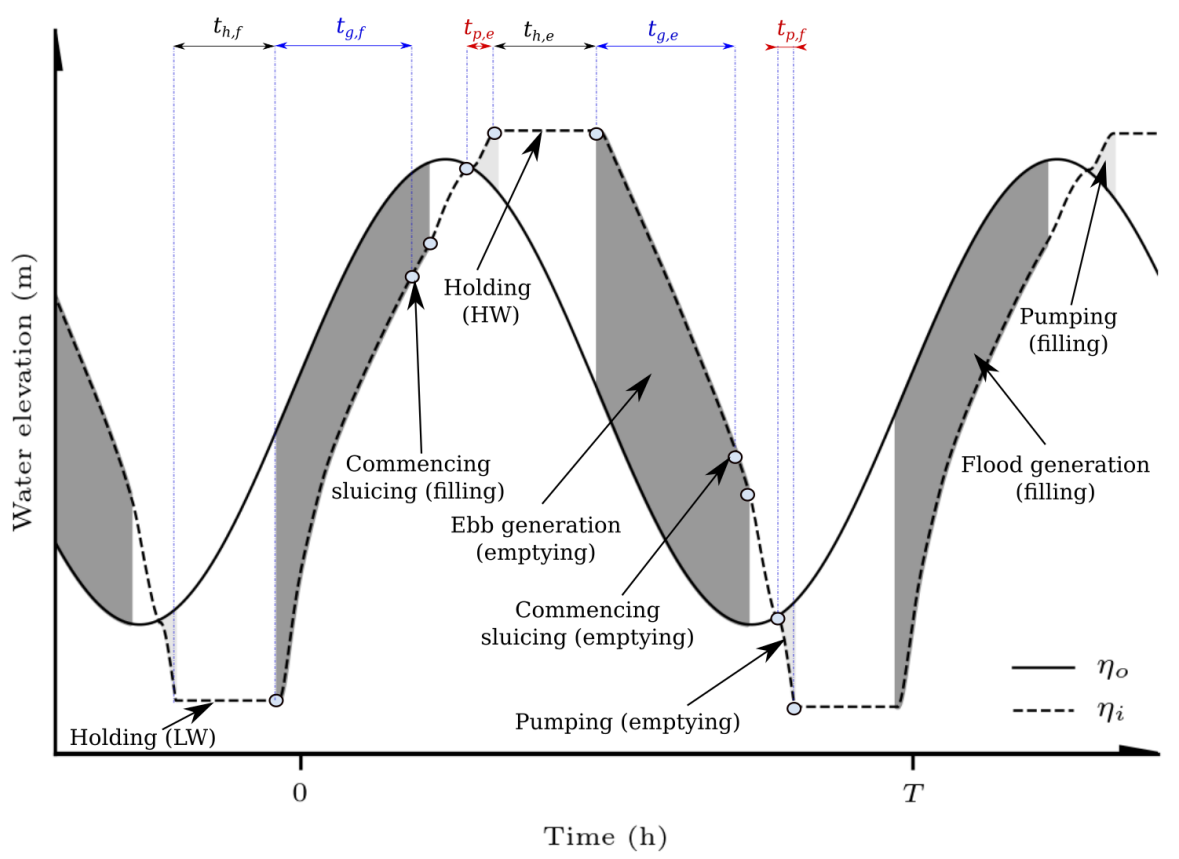

Figure 1: Generalised operation of a tidal power plant over an $M 2$ tidal period, illustrating typical modes of operation which make up $\boldsymbol{\tau}$ the vector of control parameters in our optimisation framework. The holding $\left(t_{h, f}, t_{h, e}\right)$, generation $\left(t_{g, f}, t_{g, e}\right)$ and pumping $\left(t_{p, f}, t_{p, e}\right)$ periods can be controlled to shift the operation for a limited amount of time, as constrained by the tidal signal and the capabilities of the hydraulic structures.

The efficiency of tidal power plants in harnessing the available potential energy during a given tidal cycle is heavily dependent on the control of the constituent hydraulic structures [14, 15. A generalised illustration of how a plant can be regulated is presented in Fig. 11, where $t_{p, e}, t_{g, e}, t_{h, e}, t_{p, f}, t_{g, f}, t_{h, f}$ are the main control variables that essentially dictate the operation strategy for a given tidal cycle $i$. The first subscripts refer to pumping, generating and holding respectively, while the second refers to the ebb and flood tide respectively.

In order to simulate the operation of such sequences in time, it is essential to parametrise the behaviour of turbines and sluice gates. The flows through these, and hence into and out of the impoundment, are driven by the water head difference $H=\eta_{o}-\eta_{i}$ (see Fig. 1) developed between the two sides of the structure. In our implementation the sluice gate flows are determined 
through the orifice equation, with the turbine behaviour based upon the hill chart parametrisation of Aggidis and Feather [16. Details on the formulations used in our operational modelling are expanded in Angeloudis et al. [5] and are omitted here for brevity.

\subsection{Operation modelling}

Models of tidal power plant operation essentially simulate the evolution of the water levels within the impounded (inner) body of water and the open sea through time, calculating information about the power plant performance. Hydraulic structures connect the two water bodies ensuring, at a minimum, the conservation of continuity within the impoundment [17.

\subsubsection{Zero-dimensional modelling}

A 0-D model simplifies the process of simulating a tidal power plant by assuming that the introduction of the impoundment and the operation of the constituent hydraulic structures have a negligible effect on the external water elevations. The evolution of the external $\eta_{o}(t)$ is thus purely an input to the model in the form of a time series derived from the relevant tidal constituents or observed data. The model can then calculate the head difference $H$ (Fig. 1) to determine the volume exchanged between the impounded area and the open sea. The volume exchange determines the internal water level $\eta_{i}$ for the subsequent timestep, ensuring mass (or volume, for a constant density) conservation. Mathematically the process is based on a finite difference method expressed through the equation:

$$
\frac{d \eta_{i}}{d t}=\frac{Q_{s}(m, H, t)+Q_{t}(m, H, t)+Q_{\text {in }}}{A_{s}\left(\eta_{i}\right)},
$$

where $A_{s}\left(\eta_{i}\right)$ is a site-specific function for the wetted surface area of the tidal range structure (in $\mathrm{m}^{2}$ ). $Q_{s}$ and $Q_{t}$ are the sluice and turbine flow rates as predicted by the hydraulic structure parametrisations respectively [5]. It is assumed that $\eta_{i}$ remains constant across the impoundment [13] and $Q_{\text {in }}$ accounts for inflows into tidal range projects other than the defined hydraulic structures, such as from rivers or outfalls.

\subsubsection{Two-dimensional modelling}

The drawbacks of $0-\mathrm{D}$ modelling in neglecting the impact of tidal power plant presence and operation on local and regional hydrodynamics can be quite extensive depending on the site characteristics [18. The 0-D assumption of a constant $\eta_{i}$ can equally become a short-coming for larger schemes that feature intertidal areas and a varying bathymetry. In addition, site-specific information is required on tidal constituents in order to generate the outer elevation time series used as an input in 0-D modelling.

Regional coastal ocean models can be used to predict the flow elevations, velocities and even the altered outer tidal constituents from the introduction of coastal infrastructure. In this case, we use Thetis, a (2-D and 3-D) flow 
solver [19] for simulating coastal and estuarine flows. The model is implemented by solving the non-conservative form of the nonlinear shallow water equations within the Firedrake finite element Partial Differential Equation (PDE) solver framework 20] which has already been employed for tidal range energy hydrodynamic impact assessments [21, 5].

In the case of tidal power plant hydrodynamic simulations, the representation of the turbines and sluice gates is implemented according to a flux-based method using the principles of domain decomposition [17. Flux values are determined at each time step through the same operational algorithm as in the 0 -D modelling. Water elevations are sampled at locations in the computational domain adjacent to turbines and sluice gates to determine the localised $\eta_{o}$ and $\eta_{i}$ and calculate $H$.

\subsection{Operational Optimisation}

Most renewable energy technologies are primarily concerned with the manner that energy is extracted from nature with limited control over the actual timing of this extraction. In contrast, tidal range energy alongside hydro power form an exception since there is the additional issue of when power should be produced for maximum utility. The temporal generation flexibility of tidal range energy is a result of the capability to vary the length of individual operation modes (Fig. 1) so that an overall objective is achieved, such as plant energy output maximisation. The duration of certain modes are considered our decision variables and are stored in a vector $\tau$.

\subsubsection{Fixed control parameter optimisation}

Assuming the operational control vector $\boldsymbol{\tau}$ is fixed, i.e. its entries take the same values for all tidal cycles over the simulation time $\left(t_{s}\right)$, then the objective function to maximise energy output can be written as:

$$
\max _{\boldsymbol{\tau}} \int_{t=0}^{t=t_{s}} P(\boldsymbol{\tau}, H, t) d t,
$$

where $t$ and $H$ determine the correct mode of operation that dictates which turbine and orifice equations will be used. For use below, we denote the fixed operation parameter which maximises energy output by $\boldsymbol{\tau}_{c}$. In most tidal range energy studies to-date, fixed control parameters have been assumed [22, 23, 24].

\subsubsection{Single-Cycle Optimisation}

The magnitude of the tide varies significantly over time; for example in Swansea bay, UK, the tidal range for each cycle can vary between $2 \mathrm{~m}$ and 10 $\mathrm{m}$ over neap and spring conditions respectively. Applying the same operational control for each cycle therefore almost certainly does not yield the optimal energy output. The vector $\boldsymbol{\tau}$ can be optimised to allow each operational cycle to adapt to the variable tidal elevations; a strategy we refer to as adaptive

operation. If for notational simplicity we assume that each operational cycle is 
the same length as the $M_{2}$ period $(T \approx 12.42 \mathrm{~h})$ and the simulation spans for $n_{c}$ cycles, the problem can be formulated as:

$$
\begin{array}{ll}
\text { for } i=1: n_{c} & \\
\max _{\boldsymbol{\tau}_{i}} & \int_{t=i \times T}^{t=(i+1) \times T} P\left(\boldsymbol{\tau}_{i}, H, t\right) d t \\
\text { subject to } & \boldsymbol{\tau}_{l} \leq \boldsymbol{\tau}_{i} \leq \boldsymbol{\tau}_{u}
\end{array}
$$

where $\boldsymbol{\tau}_{l}, \boldsymbol{\tau}_{u}$ correspond to the lower and upper limits expected for the different modes of operation (see Table 2). Decomposing the problem into tidal cycle steps is a simplification that limits the total number of optimisation variables resulting in fewer iterations and shorter simulations. Hitherto we have outlined established methodologies set out in previous work [5], but now turn our attention to further improving upon this existing optimisation strategy.

\subsubsection{Two-Cycle Optimisation}

A key resulting output from optimising the operation over one tidal cycle is the inner water-level $\left(\eta_{i}\right)$ at the beginning of the next cycle. This has the potential to compromise the energy output from that subsequent cycle. A symptom of this "short-sightedness" in the single-cycle approach may have been that flood generation and pumping were frequently skipped, particularly during neap tides, in earlier applications of this methodology [5]. The algorithm could have resorted to this in an attempt to compensate for the "greedy" nature of the functional in Eq. 4. The objective functional here has been reformulated to consider the operation over an additional tidal cycle as follows:

$$
\begin{array}{ll}
\text { for } i=1: n_{c} & \\
\max _{\boldsymbol{\tau}_{i}} & \int_{t=i \times T}^{t=(i+1) \times T} P\left(\boldsymbol{\tau}_{i}, H, t\right) d t+\int_{t=(i+1) \times T}^{t=(i+2) \times T} P\left(\boldsymbol{\tau}_{c}, H, t\right) d t \\
\text { subject to } & \boldsymbol{\tau}_{l} \leq \boldsymbol{\tau}_{i} \leq \boldsymbol{\tau}_{u}
\end{array}
$$

where $\boldsymbol{\tau}_{c}$ is the fixed operational pattern obtained from the optimisation described in section 2.3.1, hence the control of the second cycle is not varied. The inclusion of the second cycle in the objective function ensures that the optimisation pays some heed to the energy output from the next cycle when varying the control of the first cycle. Figure 2 illustrates differences in the optimisation process between the single-cycle and our new two-cycle optimisation approaches. The benefit of this approach is that six variables are being optimised for as in the single-cycle approach, but at the expense of a longer 0-D simulation in the functional. In contrast, a complete two-cycle optimisation would require 12 variables to be optimised for each cycle, which can rapidly become computationally inefficient. Moreover, while the optimisation can be extended further to subsequent cycles, one must observe that practically there will be a limited foresight on the exact seaward elevations in a manner that accounts for storm 
surge effects and other influencing factors that can only be finitely predicted over shorter timescales [25.

a) Single Cycle Optimisation

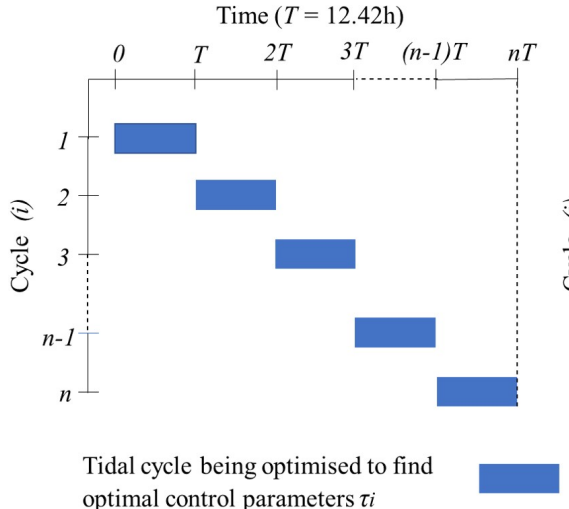

b) Two Cycle Optimisation

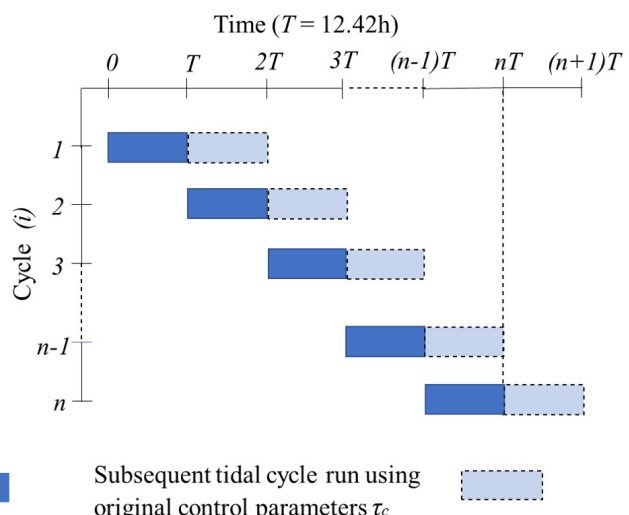

Figure 2: Illustration of the difference in how a single-cycle and two-cycle optimisation strategy is applied over $n$ cycles.

\subsubsection{Income maximisation objective}

The objective functions outlined in Eq. (4. 5) solely maximise total energy output over the entire period considered. If the energy output needs to be translated to income, the particular objectives would be underpinned by the assumption that electricity has a constant value throughout the day. In acknowledging the flexibility of the power plant generation, we adopt a functional based upon Merlin et al. [12 to formulate an optimisation problem where the overall objective becomes income maximisation.

For a single-cycle methodology this leads to the following:

$$
\begin{array}{ll}
\text { for } i=1: n_{c} & \\
\max _{\boldsymbol{\tau}_{i}} & \int_{t=i \times T}^{t=(i+1) \times T} p(t) \times P\left(\boldsymbol{\tau}_{i}, H, t\right) d t \\
\text { subject to } & \boldsymbol{\tau}_{l} \leq \boldsymbol{\tau}_{i} \leq \boldsymbol{\tau}_{u}
\end{array}
$$


and for a two-cycle approach:

$$
\begin{array}{ll}
\text { for } i=1: n_{c} & \\
\max _{\boldsymbol{\tau}_{i}} & \int_{t=i \times T}^{t=(i+1) \times T} p(t) \times P\left(\boldsymbol{\tau}_{i}, H, t\right) d t+ \\
& \int_{t=(i+1) \times T}^{t=(i+2) \times T} p(t) \times P\left(\boldsymbol{\tau}_{c}, H, t\right) d t \\
\text { subject to } & \boldsymbol{\tau}_{l} \leq \boldsymbol{\tau}_{i} \leq \boldsymbol{\tau}_{u}
\end{array}
$$

where $p(t)$ is an hourly price signal from the UK Day-Ahead spot market using data from 2017 [26], henceforth to be referred to as our price signal. Prices in the Day-Ahead spot market are set at midday for the next 24 hours starting at midnight through an auction process. They capture trends in the value of electricity that largely depend on the transient demand. Generators do not know the exact price they will receive for their power until after the auction, however they can be forecast with reasonable accuracy [27. The model herein assumes that a lagoon operator has perfect foresight over these prices in order to assess the flexibility of tidal range technology and not the bidding strategies associated with the Day-Ahead spot market.

\subsubsection{Optimisation algorithms}

As part of the analysis, we compare the application of a gradient-based optimiser as was used previously [5] and which is known to be susceptible to the issue of converging to local optima, against the results of a global optimisation algorithm. The gradient-based approach utilised here is the limited memory Broyden-Fletcher-Goldfarb-Shanno with bounds (L-BFGS-B) algorithm [28. Starting with $\boldsymbol{\tau}$ equal to $\boldsymbol{\tau}_{\boldsymbol{c}}$, in the L-BFGS-B approach the variables are individually perturbed in order to derive an approximation to the gradient of the objective function. We complement this approach with a global basin-hopping algorithm 29] that can be summarised by the following steps: random values within the acceptable ranges for $\boldsymbol{\tau}$ are selected and passed as starting points to the local L-BFGS-B algorithm. The minima/maxima obtained are either accepted, if it is the smallest/largest value found, or rejected. These steps are then iterated over a specified number of times as described by Wales [29].

Both the gradient-based and global optimisation algorithms are highly iterative, requiring numerical simulations to be run multiple times dependent on the number of variables being optimised for. The simplicity and hence efficiency of the 0-D model clearly becomes a major advantage for use within this framework. However, later sections of this work will illustrate the importance of verifying 0 -D predicted control parameters within 2-D hydrodynamics models to assess the validity of results 30 .

\subsection{Operation assessment}

Once the control parameters have been determined for each of the optimisation cases, the power output and income over the given time-frame can be 
calculated. The following simplified metrics are introduced: the percentile reduction in subsidy required, the generation value factor and the strike price equivalent. Beginning with the Fixed Control Parameter (FCP) case as a baseline case, we assume energy is sold at a fixed strike price $(S t P)$. A required annual income, $R I$, for the tidal range structure can then be calculated:

$$
R I=S t P \times E_{y r},
$$

where $E_{y r}=\int_{t=0}^{t=705 T} P\left(\boldsymbol{\tau}_{\boldsymbol{i}}, H, t\right) d t$ is the annual energy production. $R I$ can be split into the component of subsidy, $S$, and energy market income, $I$. Recent UK Government analyses have assumed the load factor will be constant for lagoons across the day [6]; in that case, the following equations can be applied to calculate the subsidy requirement:

$$
\begin{aligned}
& S+I=R I, \\
& I=E_{y r} \times \bar{p},
\end{aligned}
$$

where $\bar{p}$ is the mean Day-Ahead market price. The value factor, $v$, is a measure of the coincidence of a generation profile with times of high power price. Hirth 31] uses it to highlight the decreasing value of wind and solar generation as more capacity is installed. It is defined as:

$$
v=\bar{p}_{t} / \bar{p},
$$

where $\bar{p}_{t}$ is the average price/MWh received over a year of simulation (i.e. $t_{s}$ $=705 \mathrm{~T}$ ). For example, if the tidal power plant features a constant load factor across the year then $v$ would be equal to unity; however, if the plant is able to generate at predominantly peak price times then $v$ takes a value greater than one. Similarly, if there is a tendency to generate during low energy value periods $v$ would be below one.

The strike price is a common metric to compare different forms of generation. However, Eq. (8) does not capture the added benefit of a lagoon generating power at high value times. If $E_{y r}$ is substituted using Eq. 100 , we can rewrite Eq. (8) as:

$$
S t P=\frac{R I \times \bar{p}}{I} .
$$

The expected $I$ can be linked with the operational simulation predictions. Since load factor is not equal throughout the day, the income calculation can be rewritten as:

$$
\begin{gathered}
I=E_{y r, o p} \times \bar{p}_{t}, \\
S t P=\frac{R I}{E_{y r, o p} \times v},
\end{gathered}
$$


with $E_{y r, o p}$ being the energy output over 705 tidal cycles for our different optimisation cases. Since the UK strike prices we refer to were quoted in 2012 terms, their value in 2017 must account for the effects of inflation through the use of the CPI index. Once the optimised StP has been determined, it is converted to the equivalent 2012 monetary value [32]. The formulations above can be perceived as an oversimplification of the financial modelling required for a project of this scale, but they are designed to place our results in a broader context.

\subsection{Tidal energy case study: the Swansea Bay Tidal Lagoon}

The Swansea Bay Lagoon has been used as a case study for this work given that it was identified as a pathfinder project for a series of larger lagoons in the UK. The proposal by Tidal Lagoon Power Ltd (TLP) 33] was received favourably by the UK Government's independent "Hendry" review 8 which discussed significant potential benefits from developing lagoon-based tidal range energy generation technology. Reports state that TLP's most recent offer was an alternative strike price of $£_{2012} 92.50 / \mathrm{MWh}$ over a Contract for Difference (CfD) lasting 90 years for what is seen as a pilot project [8]. The extended CfD period relative to other forms of sustainable energy (which are typically capped at 35 years) has been justified based upon a project lifetime that could be more than 120 years [8, 34. Nonetheless, even though large-scale tidal power plants in France (La Rance) and South Korea (Lake Sihwa) have proven successful, the technology in the UK has yet to demonstrate its competitiveness relative to other forms of energy generation [6], with the UK Government recently questioning the value of the project even after the positive recommendations of Hendry 8$]$. This renders the particular application of this optimisation methodology timely, and we use the $£_{2012} 92.50 / \mathrm{MWh}$ figure that was reported in the public domain [35, 36] as a baseline example to seek to improve upon.

There are additional practicalities involved in the selection of this particular case study. As the scale of the project increases, so does its potential hydro-environmental impact [37, 38]. A similar effect occurs in the context of energy markets. Hirth [31] reports that when renewable technologies of zerovariable cost represent a reasonable proportion of generation, wholesale power price drops to between 50 and $80 \%$ of the average market value. The UK minimum electricity demand in 2017 was $16.6 \mathrm{GW}$ [26]. At this time, the $320 \mathrm{MW}$ capacity of the Swansea Bay Lagoon would only correspond to a maximum of $\approx 2 \%$ of generation. Since the scheme represents a relatively contained proportion of the country's electricity supply, it can be assumed that it would result in a negligible effect on the market price of power. We would not be able to readily make this assumption with larger or multiple tidal energy schemes, as the feedback of the power plant capacity on the energy market would need to be accounted for.

Moreover, considering the advanced stage of planning for the proposed Swansea Bay lagoon, many features have been well defined by TLP [40, 33. and studied previously in the academic literature 3 , 41. As such, turbine specifications $(16 \times 20 \mathrm{MW}$ bulb turbines of $7.35 \mathrm{~m}$ diameter $)$, the plan surface area 

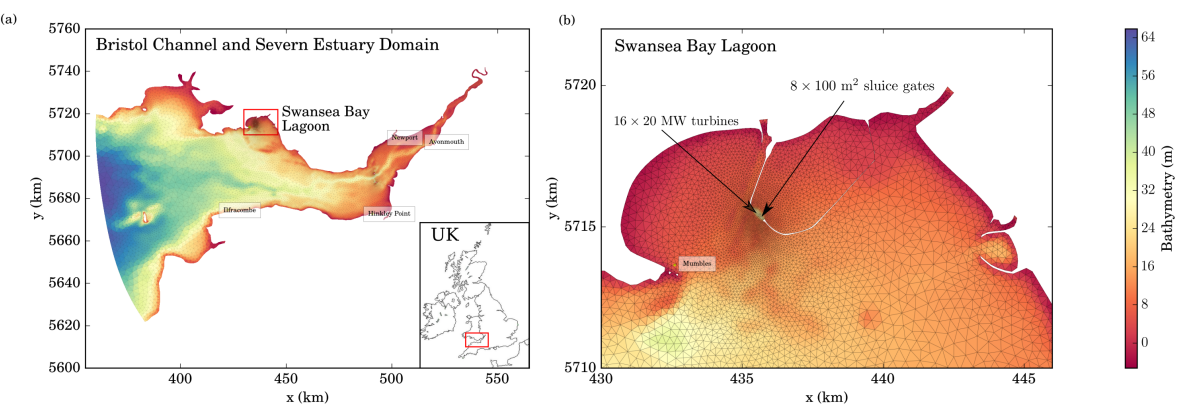

Figure 3: 2-D simulation computational domain: (a) Bristol Channel and Severn Estuary and (b) close-up to the Swansea Bay lagoon configuration. The interpolated bathymetry has been drawn from the Edina Digimap Service [39]. Coordinates are plotted using a UTM 30N geospatial projection.

of $11.6 \mathrm{~km}^{2}$ and the impoundment shape can be defined using publicly available information 42. These inputs are considered to be pre-determined and allow us to incorporate a combination of financial, environmental and technical constraints, whose details and selection are beyond the scope of this study.

Regional coastal ocean models of the Bristol Channel and the Severn Estuary were setup in Thetis. Their unstructured meshes (in Fig. 3p are generated using qmesh [43] and the models are forced using eight tidal constituents from the TPXO [4] database $\left(M_{2}, S_{2}, N_{2}, K_{1}, Q_{1}, O_{1}, P_{1}, K_{2}\right)$. The hydrodynamic model validation has been conducted by comparing tidal range predictions against observed data, with more details available in Angeloudis et al. [5]. Elevations were then sampled at the powerhouse location which allowed for extended tidal signals to be constructed through harmonic analysis of the predicted elevation time-series spanning a full lunar cycle. These signals were then used to force 0-D simulations spanning multiple years and beginning at 07:00 on $01 / 01 / 2017$, to coincide with the price signal of 2017 . The optimisation cases summarised in Table 1 were selected in order to investigate differences resulting from choice of functional formulation, optimisation algorithm and the overarching objective of the lagoon operation. 
Table 1: Optimisation cases considered. These case are characterised by the number of cycles considered in the objective functional, the algorithm used and the operational objective.

\begin{tabular}{|c|c|c|c|c|c|c|}
\hline \multirow[t]{2}{*}{ \# } & \multicolumn{2}{|c|}{ Functional duration } & \multicolumn{2}{|c|}{ Optimisation Algorithm } & \multicolumn{2}{|c|}{ Objective } \\
\hline & 1-Cycle & 2-Cycle & L-BFGS-B & Basin-hopping & Energy & Income \\
\hline 1C-BFGS-E & $\checkmark$ & & $\checkmark$ & & $\checkmark$ & \\
\hline 2C-BFGS-E & & $\checkmark$ & $\checkmark$ & & $\checkmark$ & \\
\hline $1 \mathrm{C}-\mathrm{BH}-\mathrm{E}$ & $\checkmark$ & & & $\checkmark$ & $\checkmark$ & \\
\hline 2C-BH-E & & $\checkmark$ & & $\checkmark$ & $\checkmark$ & \\
\hline 1C-BFGS-P & $\checkmark$ & & $\checkmark$ & & & $\checkmark$ \\
\hline 2C-BFGS-P & & $\checkmark$ & $\checkmark$ & & & $\checkmark$ \\
\hline 1C-BH-P & $\checkmark$ & & & $\checkmark$ & & $\checkmark$ \\
\hline 2C-BH-P & & $\checkmark$ & & $\checkmark$ & & $\checkmark$ \\
\hline \multicolumn{7}{|c|}{$\begin{array}{l}\text { Case abbreviation dictionary: } 1 \mathrm{C}=\text { Single-cycle optimisation, } 2 \mathrm{C}=\text { Two-cycle optimisation, } \\
\mathrm{BFGS}=\mathrm{L}-\mathrm{BFGS}-\mathrm{B} \text { algorithm, } \mathrm{BH}=\mathrm{Basin} \text {-hopping algorithm, } \mathrm{E}=\text { Energy maximisation objective, } \\
\mathrm{P}=\text { Income maximisation objective }\end{array}$} \\
\hline
\end{tabular}

The control variables have been constrained to vary between the limits given in Table 2 taking into account certain practical considerations. For example, the upper limit for $t_{p, f}$ is lower than $t_{p, e}$ since there is far less volume below the mean water line than above due to intertidal regions, leading to a comparatively shorter time to alter $\eta_{i}$ within the lagoon. The lower limit on all variables is set as zero, meaning effectively that modes of operation can be skipped.

Table 2: Fixed operational values and limits for the different modes of operation.

\begin{tabular}{l|c|c|cc}
\hline \multirow{2}{*}{ Control Variable } & \multirow{2}{*}{ Notation } & \multicolumn{3}{|c}{ Mode Duration $(\mathrm{h})$} \\
\cline { 3 - 6 } & & Fixed & \multicolumn{2}{|c}{ Adaptive } \\
\cline { 3 - 6 } & & $\tau_{c}[\mathbf{5}$ & $\tau_{l}$ & $\tau_{u}$ \\
\hline Flood Pumping & $t_{p, f}$ & 0.82 & 0.0 & 2.0 \\
Flood holding & $t_{h, f}$ & 1.76 & 0.0 & 4.0 \\
Flood Generation only & $t_{g, f}$ & 2.5 & 0.0 & 3.5 \\
Ebb Pumping & $t_{p, e}$ & 0.93 & 0.0 & 3.5 \\
Ebb Holding & $t_{h, e}$ & 1.79 & 0.0 & 4.0 \\
Ebb Generation & $t_{g, e}$ & 2.5 & 0.0 & 3.5 \\
\hline
\end{tabular}

\section{Results \& Discussion}

\subsection{Comparison of energy maximisation strategies}

The optimisation strategies outlined in the previous section produce the plant control parameters for each tidal cycle over an entire year (i.e 705 $T)$. Table 3 summarises the anticipated annual energy output from energymaximisation cases. Two-cycle (2C) approaches are seen to consistently lead to increased energy outputs which indicate that the single-cycle (1C) formulations through their "short-sighted" nature compromise generation in subsequent cycles. 1C strategies yield lesser annual energy output albeit still leading to an improvement over the Fixed Control Parameter (FCP) case. 
Table 3: Annual energy output $E_{y r}$ and capacity factor $C F$ for energy-maximisation cases run over 705 cycles. $E_{y r, 0}=$ Fixed control parameter $(\mathrm{FCP})$ annual energy output. $1 \mathrm{C}$ and $2 \mathrm{C}$ refers to single and two-cycle approaches; BFGS and BH refer to local and global optimisation algorithms as described in the main text.

\begin{tabular}{l|ccc}
\hline Optimisation & $E_{y r}(\mathrm{MWh})$ & $\frac{E_{y r}-E_{y r, 0}}{E_{y r, 0}} \%$ & $C F$ \\
\hline FCP & 558,448 & - & $19.92 \%$ \\
1C-BFGS-E & 613,091 & $9.8 \%$ & $21.87 \%$ \\
2C-BFGS-E & 630,308 & $12.9 \%$ & $22.49 \%$ \\
1C-BH-E & 648,129 & $16.1 \%$ & $23.12 \%$ \\
2C-BH-E & 656,051 & $17.5 \%$ & $23.40 \%$ \\
\hline
\end{tabular}

Fig. 4 presents holding and pumping control variables $\left(t_{h, e}, t_{h, f}, t_{p, e}, t_{p, f}\right)$ relative to the tidal range. Agreement can be observed between Fig. 4a, $\mathrm{b}$ as the tidal range exceeds 7 metres. In contrast, Fig. 4k,d demonstrates significantly different trends in the optimised lagoon operation, even though both strategies yield notable energy gains. There is a tendency for single-cycle functionals to push parameters to the limits, either by skipping a mode altogether, or by reaching the upper bounds in terms of duration (Table 2). For example, the control variables obtained with the $1 \mathrm{C}-\mathrm{BFGS-E}$ approach reach the adaptive operation bounds 82 times in total, with the majority of these instances occurring during neap tides. The optimisation algorithm tends to maximise generation from ebbing tides, hence $t_{p, e}$ and $t_{g, e}$ periods are frequently pushed to their limits. Pumping at flood tide is skipped (i.e. $t_{p, f}=0.0$ ) in 39 out of the 705 tidal cycles simulated, in similarity with previous observations using this particular scheme [5. This is partly attributed to the reduced efficiency assumed for flood generation due to the turbine orientation, and the reduced $A$ caused by intertidal effects, but also tidal asymmetry effects in the water elevation signal. Nevertheless, setting $t_{p, f}=0.0$ is often accompanied by extended $t_{h, f}$ values to compensate for the lack of pumping while still generating some power. This pattern is self-perpetuating on the flood tide until the tidal range gradually increases during the transition to a spring tide. Applying the basinhopping algorithm accentuates the greedy nature of single-cycle optimisations, with the 1C-BH-E case skipping flood pumping 124 times and converging to the maximum values of $t_{g, e}$ in $274 / 705$ cycles.

In contrast, for $2 \mathrm{C}$-BFGS-E there are only six instances where a parameter is pushed to the bounds. Likewise there are only 88 instances where $2 \mathrm{C}-\mathrm{BH}-\mathrm{E}$ is constrained compared to the 474 of $1 \mathrm{C}-\mathrm{BH}-\mathrm{E}$. The addition of a second cycle to the optimisation functional appears to favour a more balanced operation between flood and ebb periods. We measure this through calculating the mean of each control variable (over all tidal ranges), then comparing the ratio of the flood to ebb generation parameter averages. For 1C-BFGS-E the ratios of $\overline{t_{p, e}}: \overline{t_{p, f}}$ and $\overline{t_{h, e}}: \overline{t_{h, f}}$ were 1.38 and 1.15 respectively, indicating significantly more time is spent on increasing $H$ for ebb generation, whereas for 2C-BFGS-E these ratios are 1.045 and 0.997 .

There are two observable differences between L-BFGS-B and BH optimi- 


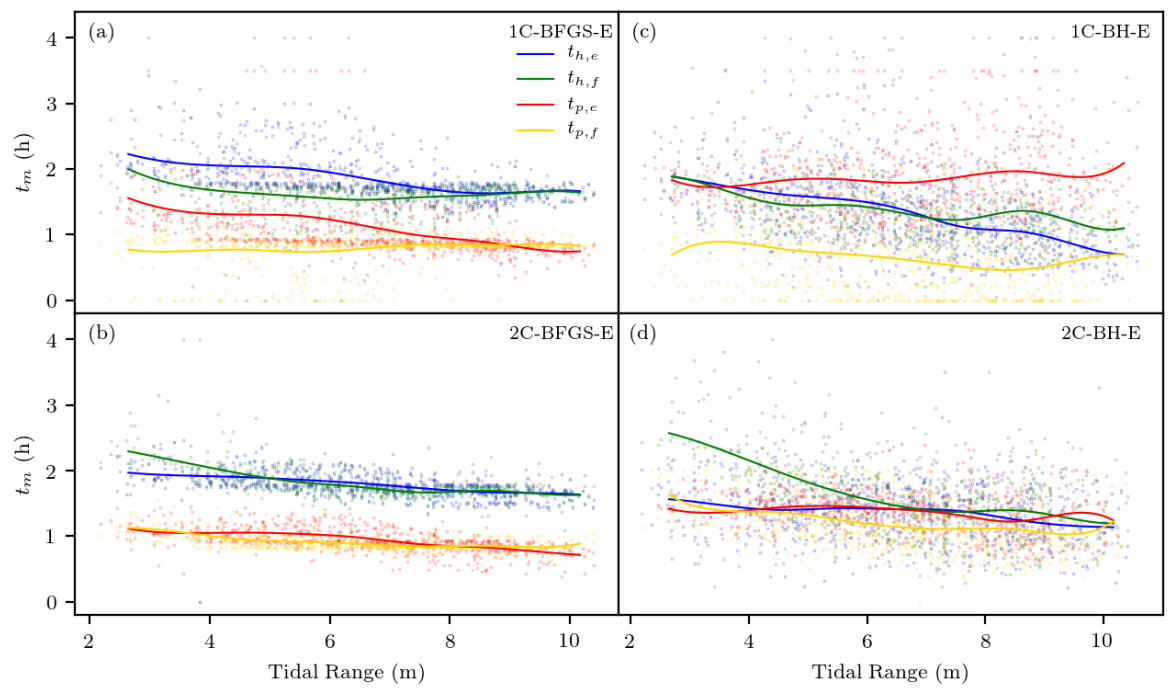

Figure 4: Holding and pumping duration for each tidal cycle for the four energy maximised optimisations. Consists of a scatter plot for each parameter, overlaid with a trendline.

sation. Firstly, BH optimisation makes a far more aggressive use of pumping, with $2 \mathrm{C}-\mathrm{BH}-\mathrm{E}$ on average spending an additional $43 \mathrm{~min}$ per tidal cycle pumping compared to $2 \mathrm{C}-\mathrm{BFGS}-\mathrm{E}$. For $1 \mathrm{C}-\mathrm{BH}-\mathrm{E}$, ebb pumping is on average the longest non-generating time period. In L-BFGS-B optimisation results, pumping periods are the shortest operating modes. Secondly, BH results feature a greater spread in the converged parameters, e.g. $t_{p, f}$ has a standard deviation of 0.13 and 0.38 for 2C-BFGS-E and 2C-BH-E respectively. Increased pumping use and greater variability in the parameters are a consequence of the stochastic nature of the $\mathrm{BH}$ algorithm that makes a greater utilisation of the parameter space. Unlike L-BFGS-B, the BH optimisation approach more broadly explores the parameter space and is thus less constrained by local minima with this flexibility delivering even better results.

\subsection{Comparison of Income and Energy Maximisation}

As introduced previously, the objective of an income maximisation based strategy is to promote generation during high-demand periods. Fig. 5 illustrates how changing the operational objective in this respect influences the internal water level $\eta_{i}$, and power output $P$. Peak prices from 17:00 - 18:00 pm on a typical January evening, as seen in the figure, offer a strong financial incentive to generate during these times. Hence the tidal power plant is encouraged to shift the timings of its operation in order to maximise income. The gains realised from selling electricity at peak prices often outweighs the potentially compromising effect on either the preceding or subsequent cycles. The previous 


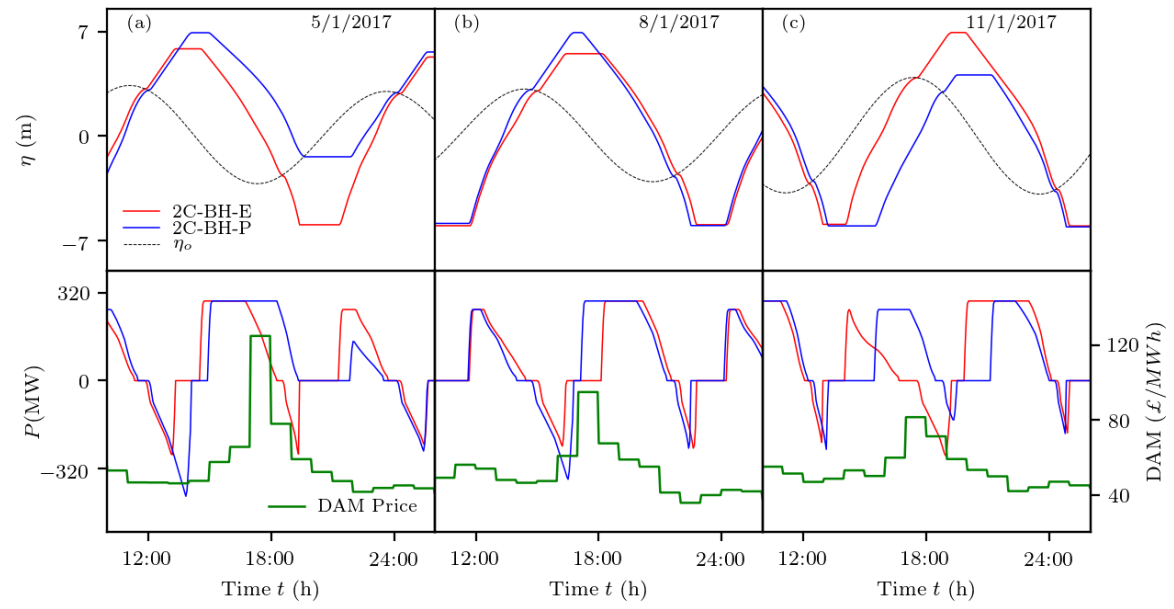

Figure 5: Comparison of the effects of income (2C-BH-P) against energy (2C-BH-E) maximisations on $\eta_{i}$ (the water level inside the lagoon) and the power output of the tidal power plant. $\eta_{o}$ is the external water level. The figures present three examples of income optimisation (blue line) (a) delaying ebb generation, (b) initiating ebb generation earlier, and (c) delaying flood generation to generate during periods of increased demand. It does this to maximise income from the Day Ahead Power Markets (DAM) which capture the variable value of electricity.

energy optimised operation is, however, ignorant of the commercial value of the electricity produced, as illustrated in Fig. 55 where the 2C-BH-E strategy initiates pumping at the evening peak price, potentially affecting the security of an electricity system.

The income optimisation strategy is, in contrast, fully aware of the transient electricity value. Income-based generation does not prioritise energy maximisation and results in an overall lower total energy output. As such, the benefit of shifting generation earlier or later is dependant on the price difference between the average price of the operational cycle and the peak price. Accordingly, if there is a pronounced price spike, the income optimisation will aim to shift generation further away from the energetically optimum operation and could theoretically (given the right price signal) be available at any time of the day. High peak prices can incentivise increased pumping to store energy from a low price time to be used during a high priced time as can be clearly seen in Fig. 5 a. This function demonstrates how tidal power plants could function along similar principles as pumped storage facilities, such as the Dinorwig pumped-storage scheme [45] in North Wales, UK. Generation cannot be sustained indefinitely, thus if there are extended periods of high price then the lagoon will seek to maximise energy output since there is no value in shifting generation over that period. 
Table 4: Quantification of the difference in total energy output, annual energy market income $I$, and the financial metrics from section 2.4 which are the value factor, $v$, the strike price equivalent, $S t P$, and percentage change in subsidy requirements, $\% \Delta S$, where $\Delta S=\frac{S-S_{\text {Baseline }}}{S_{\text {Baseline }}}$.

\begin{tabular}{l|c|cc|ccc}
\hline \multirow{2}{*}{ Optimisation } & Energy & \multicolumn{2}{|c|}{ Income } & \multicolumn{3}{c}{ Financial Metrics } \\
\cline { 2 - 7 } & $E_{y r}(\mathrm{MWh})$ & $I(£)$ & $\%$ Inc. & $v$ & $\% \Delta S$ & $S t P\left(£_{2012} / \mathrm{MWh}\right)$ \\
\hline Baseline & 558,448 & $25,307,188$ & - & 1.000 & - & 92.50 \\
FCP & 558,448 & $25,049,732$ & $-1.02 \%$ & 0.990 & $0.8 \%$ & 93.45 \\
2C-BFGS-E & 630,308 & $27,858,573$ & $10.08 \%$ & 0.975 & $-8.2 \%$ & 84.03 \\
2C-BH-E & 656,051 & $28,755,354$ & $13.63 \%$ & 0.967 & $-11.1 \%$ & 81.41 \\
2C-BFGS-P & 611,417 & $29,155,484$ & $15.21 \%$ & 1.052 & $-12.4 \%$ & 80.29 \\
2C-BH-P & 630,076 & $31,275,236$ & $23.58 \%$ & 1.095 & $-19.2 \%$ & 74.85 \\
\hline
\end{tabular}

The Baseline case in Table 4 assumes electricity generated by FCP is sold at the average market price, hence the value factor is exactly unity. All other cases sell electricity into the DAM markets. A common result observed for the FCP and energy optimisation approaches is the value factor of tidal power. If generation is not made sensitive to the energy market, the value factor is observed to be slightly less than unity, indicating that tidal lagoon power in Swansea Bay does not naturally generate when prices are high. This explains why the FCP case increases the subsidy and strike price relative to the Baseline (which assumes that $v=1$ ). It could be argued that this is merely a statistical inconsistency, but there is in fact a physical explanation.

A relationship emerges between the solar day and the tidal range of a given cycle (Fig. 6). Due to the exact 12 hour period of the $S_{2}$ solar constituent [46], there is a trend whereby spring and neap tides occur at certain times of day 9. Given the relationship between potential energy and tidal range, for energy optimisation the capacity factor $(C F)$ can be seen to be on average greater approximately four hours after spring tides. For the Swansea Bay location, spring tide peaks occur broadly concurrently with evening peak prices. Therefore the average $C F$ is not naturally high at times of peak price for the energy optimisation case. This can be observed in the 2C-BH-E line of Fig. 6. The effect of this relationship between power output and the $S_{2}$ constituent is location specific, and other proposed projects might naturally have a higher average $\mathrm{CF}$ at peak times.

Changing the optimisation objective to income has a noticeable effect on tidal range power plant income and energy output as seen in Table 4 . Both income-based optimisation cases receive significantly more income from each unit of energy sold, as indicated by the $v$ values. 2C-BH-P has a higher $v$ than 2C-BFGS-P as the BH approach is adept at finding maxima in a complex optimisation surface in this multi-objective problem, generating more electricity during peak price times.

Compared to the energy output of $2 \mathrm{C}-\mathrm{BH}-\mathrm{E}, 2 \mathrm{C}-\mathrm{BH}-\mathrm{P}$ receives over $12 \%$ more income per unit of energy sold, but generates $5 \%$ less power overall. This still yields nearly a $10 \%$ increase in annual income. Figure 6p illustrates how income optimised operation generates far more power at certain times of the 


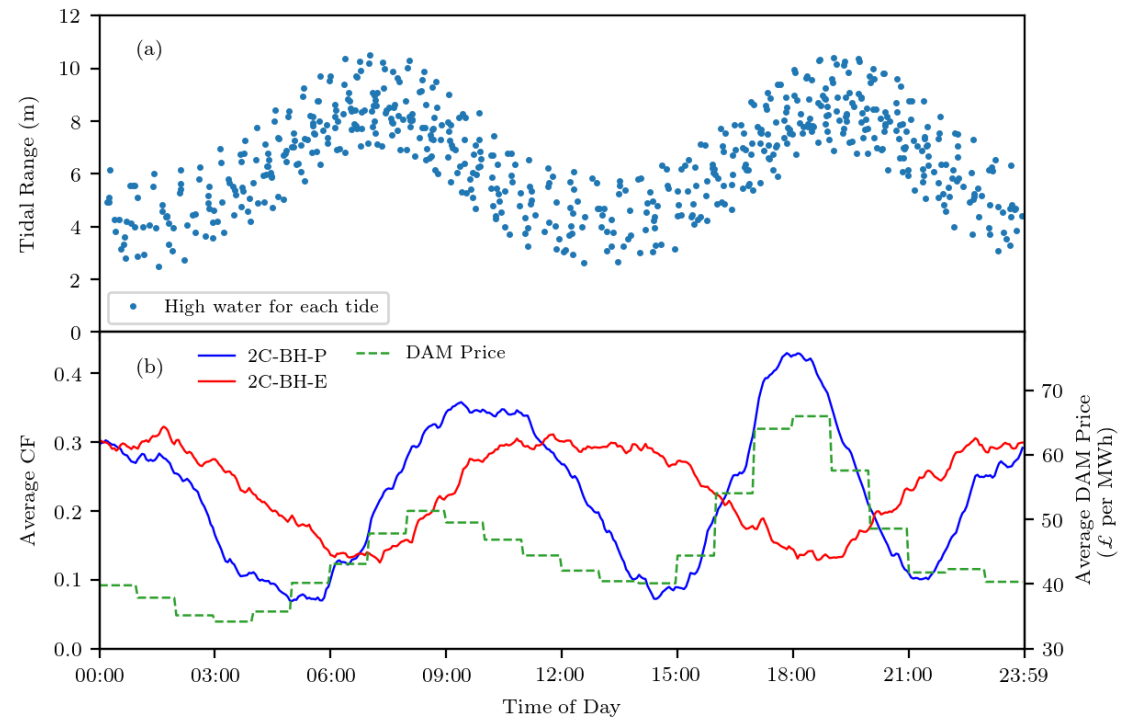

Figure 6: (a) Tidal range against high tide tie at the Swansea Bay tidal lagoon site for 705 tidal cycle. In (b) the primary $y$ axis is a plot of mean Capacity Factor, $C F$, across the day for a given control strategy. The strategies were optimised over 705 tidal cycles. The secondary $y$ axis is the mean Day Ahead Market price $(D A M)$ for a day in 2017. Note this plot does not factor in daylight savings time.

day. It can be seen to target the evening peak price times between 17:00 - 19:00 relative to the mean $C F$ over a year of operation. A significant proportion of generation still occurs overnight $(21: 00-4: 00)$ when demand for and prices of electricity are low. Thus a tidal range structure cannot be seen solely as a "peaking power plant" for when prices are high. Nonetheless, under 2C-BH-P operation the plant generates over three times as much power at the evening peak than the early hours of the morning when demand is low. The increased income generated in the $2 \mathrm{C}$-BH-P case results in a decreased subsidy requirement of $20 \%$ relative to the FCP case. The StP figure quoted captures the impact of this subsidy reduction in rendering tidal range structures more competitive relative to other renewable energy technologies. However, the benefits of such a flexible operation are not taken into account within the current Contract for Difference (CfD) regulatory framework. This is because a CfD isolates renewable generators from the wholesale electricity markets, merely motivating the maximisation of energy output. Aside from cost, there are other potential benefits of income optimised operation, as discussed in the next section. 


\subsection{The potential role of Tidal Range Generation}

Fig $7 \mathrm{~A}$ is based on the optimised operation results over the annual period of 2017 . For Fig. $7 \mathrm{~b}$ operation was optimised over five years $(705 \times 5$ cycles $)$, with the 2017 price signal assumed to be identical for each of these in order to represent the daily and seasonal energy price trends subject to varying tidal conditions over an extended period of time. For the latter, while we could have used historical energy market data, the UK energy system has fundamentally changed since 2013 when the simulation would have began. During 2013 coal was still representing $36 \%$ of the energy mix and renewables occupied less than $15 \%$ [47. In 2017 coal was reduced to $6.3 \%$, with renewables accounting for almost 30\% 48. Running a five year simulation was primarily used to increase the number of high value price hours seen in Fig. 7, reducing the probability that the trends seen in Fig $7 \mathrm{a}$ are coincidental. In 2017 there were nine instances of the Day Ahead market price being above 120/MWh, by using looping we increased this to 45 . Fig. 7 a suggests volatility in the $2 \mathrm{C}-\mathrm{BH}-\mathrm{P}$ line due to the limited price points with higher values; longer simulations suggest a smoother trend (Figure. $7 \mathrm{~b}$ ) as the longer simulation gives more accurate averages.

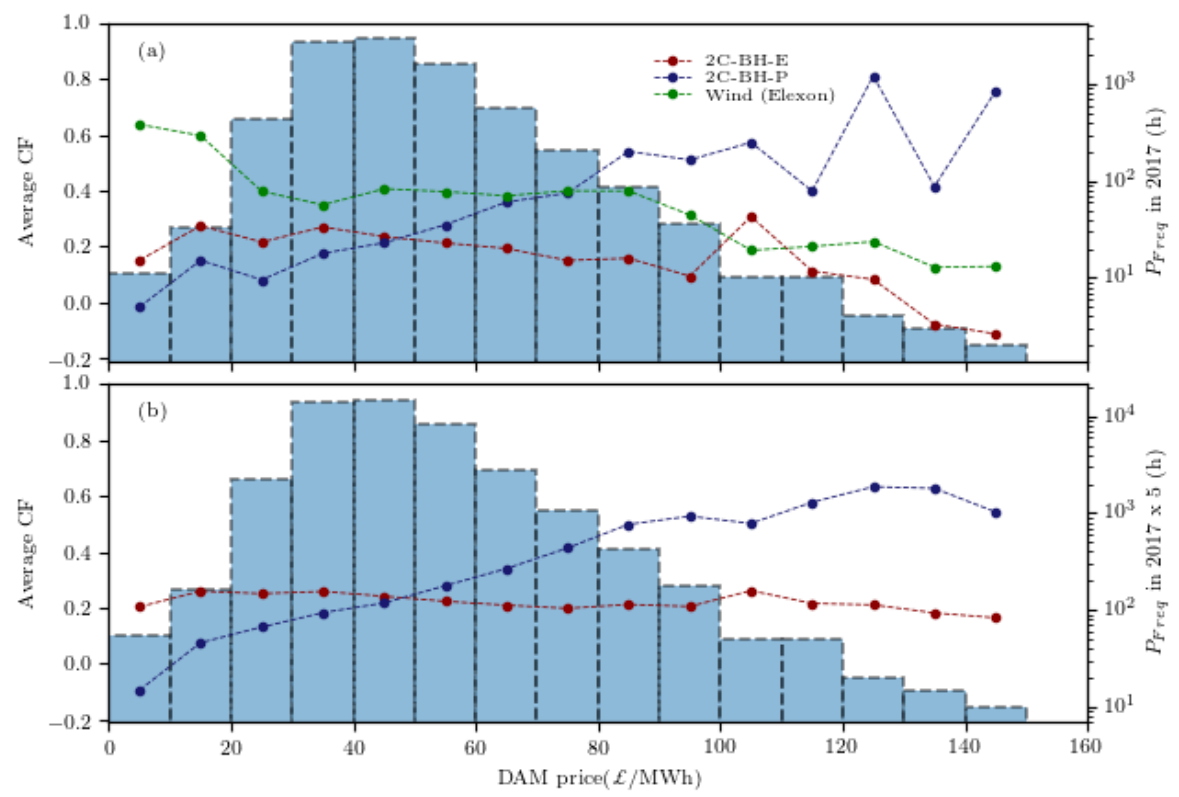

Figure 7: Left axis is the average capacity factor $(\mathrm{CF})$ against the Day Ahead market price for a) 705 tidal cycles which is approximately a year, b) $705 \times 5$ tidal cycles $\approx 5$ years to smooth trends. The secondary $y$ axis on both plots corresponds to the histogram, counting the frequency of a given price range occurring over the simulation timespan.

Energy Market prices are set through the intersection of supply and demand curves. Hence, the highest prices occur when demand is high and supply from 
zero-marginal cost renewables is low [49]. By plotting capacity factor against the price of power, seen in Fig. 7, the relationship between income and energy optimised results with the electricity price can be seen. The figure also shows the relationship between transmission connected wind [50] and price, illustrating that high wind power output effectively causes the wholesale power price to drop, confirming observations made by Hirth 31 .

As expected, energy-optimised operation displays lower capacity factors at high price times due to the timing of the spring tides at the Swansea Bay location of this study. In contrast, $2 \mathrm{C}-\mathrm{BH}-\mathrm{P}$ has a distinct upwards relationship with price, targeting the highest price times. Importantly, this could help compensate for the lack of wind power during these times, implying there could be a complimentary relationship between tidal range and wind power. Tidal range power plants would be competing against flexible thermal generation rather than exacerbating the price volatility issues associated with high penetrations of inflexible renewables supported by fixed subsidies [49]. Since price is correlated with the amount of thermal generation and thus the carbon intensity $\left(g \mathrm{CO}_{2} / K W h_{e}\right)$, by generating at the high price times, the lagoon can displace worse fossil fuel generators such as coal and open-cycle gas turbines [51].

The results indicate that the $|C F|$ of the tidal range power station generating in times of high price is far greater for $2 \mathrm{C}-\mathrm{BH}-\mathrm{P}$ than $2 \mathrm{C}-\mathrm{BH}-\mathrm{E}$. If the correct regulatory framework is not in place, energy-maximised operation could result in pumping occurring at high demand times (Fig. 7). By making projects sensitive to the energy market price, they generate far more power when demand is high, rather than low. Given the reliability of the tides and energy storage opportunities available with the income-optimised operation strategy, tidal range energy can help ensure security of supply for the electricity system in a way that some alternative renewable technologies can not due to their intermittency and lack of predictability.

\subsection{Numerical model limitations and sensitivities}

Table 5: Energy output and Income for the Swansea Bay Lagoon Case study for both the 0-D model and 2-D model (Thetis). Models have been run over 30 days, or 58 M2 tidal cycles, beginning on $01 / 01 / 2017$. The third $\frac{2 D-0 D}{0 D} \%$ is a measure of the percentage difference between the two models.

\begin{tabular}{l|ccc|ccc}
\hline \multirow{2}{*}{$n_{c}=58$} & \multicolumn{3}{|c}{ Energy Output $(\mathrm{MWh})$} & \multicolumn{3}{c}{ Income $(£)$} \\
\cline { 2 - 7 } & $0-D$ & $2-D$ & $\frac{2 D-0 D}{0 D} \%$ & $0-D$ & $2-D$ & $\frac{2 D-0 D}{0 D} \%$ \\
\hline FCP & 46,692 & 49,222 & $5.4 \%$ & $2,449,108$ & $2,554,345$ & $4.30 \%$ \\
1C-BFGS-E & 50,306 & 54,454 & $8.2 \%$ & $2,663,316$ & $2,854,100$ & $7.2 \%$ \\
2C-BFGS-E & 51,204 & 55,362 & $8.1 \%$ & $2,687,689$ & $2,906,969$ & $8.2 \%$ \\
1C-BH-E & 52,476 & 53,170 & $1.3 \%$ & $2,772,843$ & $2,782,790$ & $0.4 \%$ \\
2C-BH-E & 53,106 & 54,977 & $3.5 \%$ & $2,779,425$ & $2,844,468$ & $2.3 \%$ \\
\hline 2C-BFGS-P & 48,732 & 54,050 & $10.9 \%$ & $2,782,868$ & $3,010,707$ & $8.2 \%$ \\
2C-BH-P & 50,671 & 48,289 & $-4.7 \%$ & $3,038,296$ & $2,882,103$ & $-5.1 \%$ \\
\hline
\end{tabular}


The operational model results presented so far utilised a 0-D model on the grounds of computational simplicity/efficiency as we considered annual and multi-year simulations (e.g. as in Fig. 7). Earlier studies have already demonstrated that due to interactions between the regional scale flow, the impoundment and the turbines are far better captured in 2-D, deviations can exist between 0-D and 2-D model results [5, 11. Nonetheless, the valuable predictive ability is still contained within the 0-D approach. Month-long 2-D model operational simulations were carried out in Thetis to investigate whether the improvements in energy output and income are realisable once we better acknowledge the extra physical processes associated with regional scale tidal dynamics. As such, control parameters stemming from the 0-D optimisation were imposed for the Swansea Bay tidal lagoon operation within the 2-D hydrodynamic simulations.Based on these control parameters the power plant in 2-D aimed to preserve the same holding and pumping periods as well as initiating sluicing when necessary during the generation for each tidal cycle. However, turbines and sluice gates in the 2-D model are still constrained in their operation by the head difference value. This can lead to a difference in the elevations and flows that were originally predicted within the 0 - $\mathrm{D}$ model. This has been accounted for to ensure that the hydraulic structures are not performing beyond their capabilities.

Table 5 summarises how simulating in a more complex hydrodynamic environment does not have a consistent effect on the anticipated energy output. Distinct shifts can be observed in Fig. 8 regarding the timings of operations, attributed to the non-linear interactions which occur once more physical processes are included. In improving the performance of the strategies and given that the timing of generation becomes crucial relative to the price signal, control parameters used in the 2-D model were corrected by averaging the holding period duration imposed and the actual time that ebb/flood generation commenced in the 0-D model for the corresponding tidal cycle. This correction encouraged generation to commence as close to the equivalent timings predicted through the 0 - $\mathrm{D}$ based optimisation, without significant changes to the holding periods that were determined as control parameters. For most cases (e.g. 2C-BFGS-P in Fig. 8(a)), 2-D results closely follow the 0-D predictions demonstrating the value of the operational optimisation leading to a performance that is superior to what was initially expected from the FCP case. However, in the case of 2C-BH-P (Fig. 8(b)) as the $\mathrm{BH}$ algorithm promotes extreme variations in the power plant control, the 2-D model fails at times to consistently reproduce the head difference evolution driving the turbines and sluice gates. This results in deviations in the energy production of the plant. Table 5 summarises how the $2 \mathrm{C}-\mathrm{BH}-\mathrm{P}$ control parameters correspond to a $\approx 5 \%$ reduced income and energy relative to that predicted from the 0-D analysis.

For the particular period selected it is clear that the optimisation overall benefits both income and energy objectives, with the 0-D control parameters also in general leading to an improved performance once applied to the 2-D model power plant operation. In addition, for this particular case 2-D results tend to yield greater energy outputs relative to 0-D results from earlier studies 
[5]. The latter is considered coincidental as per the month selected and would not be expected to be generally true for larger schemes where the hydrodynamic impact would lead to greater deviations from the 0-D model. However, even for this relatively small-scale scheme where regional hydrodynamic impacts are expected to be low, differences between $0-\mathrm{D}$ and 2-D hydrodynamic results do appear. As such, future work should consider the full integration of a 2-D hydrodynamic model within the optimisation functional; this would be far more computationally challenging but has been successfully achieved in the context of tidal turbine array optimisation [52, 53, 54].
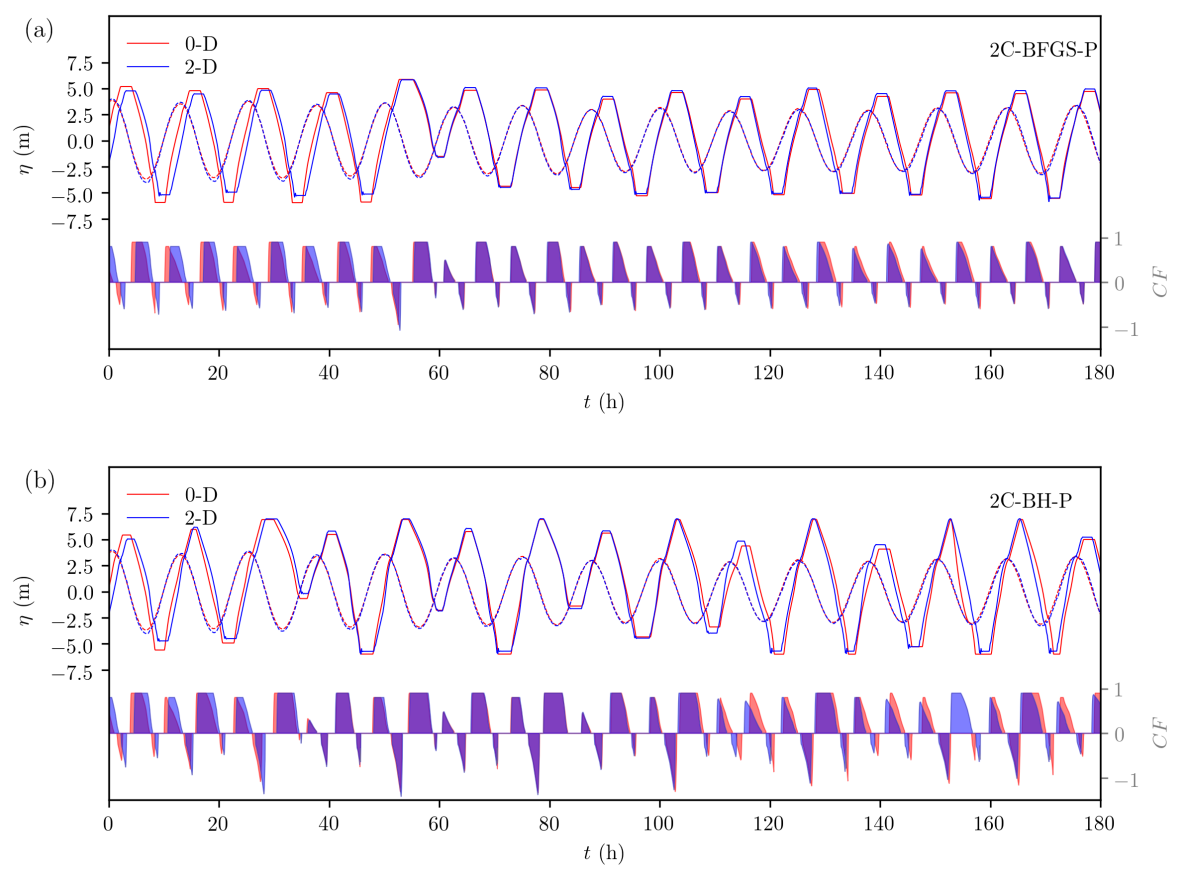

Figure 8: Comparison of the differences between $\eta_{i}$ and Capacity Factor $(C F)$ for 0D and 2D simulations over 180 hours of operation for the following cases: (a) 2C-BFGS-P; (b) 2C-BH-P.

There are other limitations to this work in assuming that an operator has perfect foresight over power prices, that the lagoon operation itself has no impact on power prices, and that electricity can be bought and sold at exactly the market price. The larger the lagoon modelled, the greater the error in these assumptions become. Nevertheless, these issues do not devalue the potential flexibility which tidal range structures might be able to offer the UK power system. 


\subsection{Methodology capabilities \& 8 applications}

The methods outlined here represent a development in adaptive operation of tidal range power generation. The optimisation objective functional has been altered to consider the impact of the current operational cycle on the subsequent cycle to prevent some of the negative impacts of a short-termist "greedy" approach on longer term behaviour. We have also tested a global optimisation algorithm in place of the local optimisation technique presented previously [5]. These modifications both led to noticeable increases in the estimated annual energy output and would help make tidal range projects more competitive against other generation technologies, without having to change any proposed infrastructure. Crucially, we demonstrated that by introducing a new optimisation objective seeking to maximise lagoon income from the Day Ahead energy markets, both the variability of the tides and the value of electricity is taken into account. Using this approach the power plant aims to generate at peak demand times since this is when the value of electricity is highest. This significantly increases the monetary value of tidal lagoon power output, reducing the subsidy required if this flexibility is accounted for in the calculations. Further work could explore whether tidal range structures generating at peak times could reduce the need for higher carbon emitting generators, and thus evaluate the carbon emission reductions associated with different operating strategies.

There is potential to re-evaluate the role multiple tidal range projects operating flexibly could play in the UK electricity system. A previous study indicated base-load power was not achievable given the tidal phasing of potential projects in the UK [14. However, by utilising flexible operation this might be possible. The work presented here demonstrates that there might be more value in targeting generation during peak times and exploiting short-term pumped storage capabilities when there is excess renewable generation from solar, wind and other alternatives. The results presented here are specific to the Swansea Bay Lagoon, but there is potential to transfer these methodologies to other proposed tidal range schemes in order to optimise their operation and feasibility. In addition, given the relationship between the $S_{2}$ tidal constituent phase and the timing of power output, certain sites might be more favourable to develop than others in this regard.

\section{Conclusions}

This paper has proposed refinements to the process of optimising tidal range power plant operation, in particular by seeking to take advantage of the potential for partial flexibility in the generation timing. This manifests in the ability to control the duration of modes within the plant's operational cycle within certain constraints, and subject to the hydraulic structure technology capabilities installed. To achieve this, operational models of tidal power plants over short intervals of time are used within an iterative optimisation framework so that optimal control parameters can be determined. Earlier approaches focused on maximising energy output from a single $M_{2}$ tidal cycle using gradient-based 
optimisation. In this study we additionally accounted for the impact of the operational control within one tidal cycle on the subsequent cycle's performance, and also applied a basin-hopping global optimisation algorithm to gauge the potential benefits in terms of increasing the estimated annual energy output from a given design. In turn, given the transient variability of demand as well as the tides we introduced an alternative strategy that sought to operate a tidal power plant to maximise income from the Day-Ahead Energy markets, rather than solely maximising total energy output. The motivation for this was to utilise the partial flexibility present in lagoon operation to increase income by incentivising generation when the price of electricity is higher.

The study sought to optimise the operation of the proposed Swansea Bay tidal lagoon over a year, selling power into the 2017 Day-Ahead Energy markets. The specifications for the test case considered were informed by technical reports in the public domain stemming from developer and official UK government documents. Reported figures regarding the Contract for Difference were used as the baseline which we sought to improve upon using operational optimisation. Results suggest that $23 \%$ more income can be achieved annually relative to the baseline figure obtained with a uniform operation, and 10\% more than with an energy-maximisation adaptive operation strategy. Income-based optimisation results in a greater power output during the evening peak demand for electricity, when power prices within the Day-Ahead energy market are expected to spike sharply. The results suggest that this flexibility could become an asset to the UK electricity system and could help ensure security of supply whilst also reducing carbon emissions.

\section{Acknowledgements}

F. Harcourt acknowledges the support from the ScottishPower Foundation who funded the Masters degree which led to this research. A. Angeloudis acknowledges the support of the NERC Industrial Innovation fellowship grant NE/R013209/2. M. Piggott acknowledges the support of EPSRC under grants $\mathrm{EP} / \mathrm{M} 011054 / 1$ and EP/R029423/1. We would also like to thank the anonymous reviewers for their insightful comments and suggestions that helped improve the manuscript.

\section{References}

[1] S. P. Neill, A. Angeloudis, P. E. Robins, I. Walkington, S. L. Ward, I. Masters, M. J. Lewis, M. Piano, A. Avdis, M. D. Piggott, G. Aggidis, P. Evans, T. A. Adcock, A. Zidonis, R. Ahmadian, R. Falconer, Tidal range energy resource and optimization past perspectives and future challenges, Renewable Energy 127 (2018) 763-778.

[2] K. Mackinnon, H. C. Smith, F. Moore, A. H. van der Weijde, I. Lazakis, Environmental interactions of tidal lagoons: A comparison of industry perspectives, Renewable Energy 119 (2018) 309 - 319. 
[3] S. Waters, G. Aggidis, Tidal range technologies and state of the art in review, Renewable and Sustainable Energy Reviews 59 (2016) 514-529.

[4] A. Angeloudis, R. Ahmadian, R. A. Falconer, B. Bockelmann-Evans, Numerical model simulations for optimisation of tidal lagoon schemes, Applied Energy 165 (2016) 522-536.

[5] A. Angeloudis, S. C. Kramer, A. Avdis, M. D. Piggott, Optimising tidal range power plant operation, Applied Energy 212 (2018) 680-690.

[6] TLP Tidal Lagoon Programme: Summary value for money assessment, Technical Report, Department for Buisness, Energy and Industrial Strategy, UK Govermnent, 2018. URL: https://assets.publishing.service.gov.uk/government/ uploads/system/uploads/attachment\{_\}data/file/719188/ tidal-lagoon-programme-vfm-summary.pdf

[7] I. Staffell, R. Green, Is There Still Merit in the Merit Order Stack? The Impact of Dynamic Constraints on Optimal Plant Mix, IEEE Transactions on Power Systems 31 (2016) 43-53.

[8] C. Hendry, The role of tidal lagoons, Technical Report, UK Government, 2017. URL: https://hendryreview.com/.

[9] T. L. Shaw, M. J. Watson, Flexible power generation from the tides, Proceedings of the Institution of Civil Engineers Engineering Sustainability 156 (2003).

[10] G. Aggidis, D. Benzon, Operational optimisation of a tidal barrage across the Mersey estuary using 0-D modelling, Ocean Engineering 66 (2013) 69-81.

[11] N. Yates, I. Walkington, R. Burrows, J. Wolf, The energy gains realisable through pumping for tidal range energy schemes, Renewable Energy 58 (2013) 79-84.

[12] A. Merlin, P. Sandrin, J. Gres, M. Hilairet, Agra, the New Operation Model for the "La Rance" Tidal Power Plant, IEEE Transactions on Power Apparatus and Systems PAS-101 (1982) 290-294.

[13] D. Prandle, Simple theory for designing tidal power schemes, Advances in water resources 7 (1984) 21-27.

[14] R. Burrows, I. Walkington, N. Yates, T. Hedges, J. Wolf, J. Holt, The tidal range energy potential of the West Coast of the United Kingdom, Applied Ocean Research 31 (2009) 229-238.

[15] J. Xia, R. A. Falconer, B. Lin, Hydrodynamic impact of a tidal barrage in the Severn Estuary, UK, Renewable Energy 35 (2010) 1455-1468. 
[16] G. Aggidis, O. Feather, Tidal range turbines and generation on the solway firth, Ren. Energy 43 (2012) 9 - 17.

[17] A. Angeloudis, R. Falconer, S. Bray, R. Ahmadian, Representation and operation of tidal energy impoundments in a coastal hydrodynamic model, Renewable Energy 99 (2016) 1103-1115.

[18] J. Wolf, I. A. Walkington, J. Holt, R. Burrows, Environmental impacts of tidal power schemes, Proceedings of the ICE Maritime Engineering 162 (2009) 165-177.

[19] T. Kärnä, S. C. Kramer, L. Mitchell, D. A. Ham, M. D. Piggott, A. M. Baptista, Thetis coastal ocean model: discontinuous galerkin discretization for the three-dimensional hydrostatic equations, Geoscientific Model Development 11 (2018) 4359-4382.

[20] F. Rathgeber, D. A. Ham, L. Mitchell, M. Lange, F. Luporini, A. T. T. Mcrae, G.-T. Bercea, G. R. Markall, P. H. J. Kelly, Firedrake: Automating the finite element method by composing abstractions, ACM Trans. Math. Softw. 43 (2016) 24:1-24:27.

[21] C. V. M. Vouriot, A. Angeloudis, S. C. Kramer, M. D. Piggott, Fate of large-scale vortices in idealized tidal lagoons, Environmental Fluid Mechanics (2018).

[22] J. Xia, R. A. Falconer, B. Lin, G. Tan, Estimation of annual energy output from a tidal barrage using two different methods, Applied Energy 93 (2012) $327-336$.

[23] J. Zhou, R. A. Falconer, B. Lin, Refinements to the EFDC model for predicting the hydro-environmental impacts of a barrage across the Severn Estuary, Ren. Energy 62 (2014) 490-505.

[24] A. Cornett, J. Cousineau, I. Nistor, Assessment of hydrodynamic impacts from tidal power lagoons in the Bay of Fundy, International Journal of Marine Energy 1 (2013) 33-54.

[25] M. Lewis, A. Angeloudis, P. Robins, P. Evans, S. Neill, Influence of storm surge on tidal range energy, Energy 122 (2017) 25 - 36.

[26] Nord Pool Spot, N2EX Day Ahead Auction Prices, UK Hourly Power Demand, 2017.

[27] J. Andrade, J. Filipe, M. Reis, R. Bessa, Probabilistic Price Forecasting for Day-Ahead and Intraday Markets: Beyond the Statistical Model, Sustainability 9 (2017) 1990.

[28] C. Zhu, R. H. Byrd, P. Lu, J. Nocedal, Algorithm 778: L-bfgs-b: Fortran subroutines for large-scale bound-constrained optimization, ACM Trans. Math. Softw. 23 (1997) 550-560. 
[29] D. Wales, Exploring the landscape, in: Energy Landscapes, Cambridge University Press, Cambridge, 2004, pp. 283-363. URL: https://www. cambridge.org/core/product/identifier/CB09780511721724A043/ type/book\{_\}part. doi:10.1017/CB09780511721724.007.

[30] A. Angeloudis, M. D. Piggott, S. C. Kramer, A. Avdis, D. Coles, Comparison of 0-D , 1-D and 2-D model capabilities for tidal range energy resource assessments, in: EWTEC 2017, Cork, 2017, pp. 1-10.

[31] L. Hirth, The market value of variable renewables: The effect of solar wind power variability on their relative price, Energy Economics 38 (2013) 218 -236 .

[32] Low Carbon Contracts Company, Guidance on Strike Price Adjustments under Contracts for Difference and Investment Contracts, Technical Report, 2017. URL: https://lowcarboncontracts.uk/sites/default/ files/StrikePriceAdjustmentGuide-January2017.pdf.

[33] Tidal Lagoon Power Development Consent Application, http://www. tidallagoonpower.com/application-for-development-consent/, 2017. Accessed: 15-10-2017.

[34] Tidal Lagoon Power, Audit of BEIS statement on Tidal Lagoons, Technical Report, 2018. URL: http://www.tidallagoonpower.com/wp-content/ uploads/2018/07/BEIS-statement-on-Swansea-Bay-Tidal-Lagoon. pdf.

[35] S. Messenger, Tidal lagoon 'has offered government new deal on price' - BBC News, 2018. URL: https://www.bbc.co.uk/news/ uk-wales-south-west-wales-43159608.

[36] S. Messenger, Swansea Bay tidal lagoon: How the numbers were crunched - BBC News, 2018. URL: https://www.bbc.co.uk/news/ uk-wales-44601268.

[37] K. Elliott, H. C. Smith, F. Moore, A. H. van der Weijde, I. Lazakis, A systematic review of transferable solution options for the environmental impacts of tidal lagoons, Marine Policy 99 (2019) 190 - 200.

[38] R. A. Falconer, A. Angeloudis, R. Ahmadian, Modeling Hydroenvironmental Impacts of Tidal Range Renewable Energy Projects in Coastal Waters, WORLD SCIENTIFIC, 2018, pp. 1553-1574. doi 10. 1142/9789813204027_0055.

[39] Edina Digimap Service, Hydrospatial one, gridded bathymetry, http:// digimap.edina.ac.uk/marine/, 2014. , SeaZone Solutions Ltd, Online; accessed 2017. 
[40] Tidal Lagoon Power turbine technology, http://www.tidallagoonpower. com/tidal-technology/turbine-technology/, 2017. Accessed: 15-102017.

[41] S. Petley, G. Aggidis, Swansea Bay tidal lagoon annual energy estimation, Ocean Engineering 111 (2016) 348-357.

[42] A. Angeloudis, R. A. Falconer, Sensitivity of tidal lagoon and barrage hydrodynamic impacts and energy outputs to operational characteristics, Ren. Energy 114(A) (2017) 337-351.

[43] A. Avdis, A. S. Candy, J. Hill, S. C. Kramer, M. D. Piggott, Efficient unstructured mesh generation for marine renewable energy applications, Renewable Energy 116 (2018) 842 - 856 .

[44] G. D. Egbert, S. Y. Erofeeva, Efficient inverse modeling of barotropic ocean tides, Journal of Atmospheric and Oceanic Technology 19 (2002) 183-204.

[45] B. Hernen, The dinorwic pumped-storage scheme, Electronics and Power 23 (1977) 828-833.

[46] NOAA, Harmonic Constituents - National Oceanic and Atmospheric Administration Tides \& Currents, 2018. URL: https: $/ /$ tidesandcurrents $\cdot$ noaa $\cdot$ gov $/$ harcon $\cdot$ html unit $=0\{\&\}$ timezone $=$ $1\{\&\}$ id $=9455920\{\&\}$ name $=$ Anchorage $\{\&\}$ state $=A K$

[47] Department of Energy and Climate Change, Digest of UK Energy Statistics 2014, Technical Report, 2014. URL: https://assets. publishing.service.gov.uk/government/uploads/system/uploads/ attachment\{_\}data/file/342760/Press\{_\}Notice\{_\}2014\{_\}v2.pdf.

[48] Departments for Business Innovation and Industrial Strategy, Digest of UK Energy Statisitcs, Technical Report, 2018. URL: www.semo.com/ marketdata/pages/energysettlement.aspx.

[49] K. De Vos, Negative Wholesale Electricity Prices in the German, French and Belgian Day-Ahead, Intra-Day and Real-Time Markets, The Electricity Journal 28 (2015) 36-50.

[50] Elexon, Historic Generation by Fuel Type, 2017. URL: https://www. elexonportal.co.uk/article/view/216?cachebust=y4tozc1xig

[51] I. Staffell, Measuring the progress and impacts of decarbonising British electricity, Energy Policy 102 (2017) 463-475.

[52] S. Funke, P. Farrell, M. Piggott, Tidal turbine array optimisation using the adjoint approach, Renewable Energy 63 (2014) 658-673.

[53] D. M. Culley, S. W. Funke, S. C. Kramer, M. D. Piggott, Integration of cost modelling within the micro-siting design optimisation of tidal turbine arrays, Renew. Energy 85 (2016) 215-227. 
[54] D. Culley, S. Funke, S. Kramer, M. Piggott, A surrogate-model assisted approach for optimising the size of tidal turbine arrays, International Journal of Marine Energy 19 (2017) 357 - 373. 